\title{
Healthcare and Financial Decision Making and Incident Adverse Cognitive Outcomes among Older Adults
}

Christopher C. Stewart PhD'1, Lei Yu PhD,2,3, Robert S. Wilson PhD ${ }^{2,3,4}$, David A. Bennett $\mathrm{MD}^{2,3}$, Patricia A. Boyle $\mathrm{PhD}^{2,4}$.

1. Department of Neurology, Indiana University School ofMedicine, Indianapolis, Indiana;

2. Rush Alzheimer's Disease Center, RushUniversity Medical Center, Chicago, Illinois;

3. Department of NeurologicalSciences, Rush University Medical Center, Chicago, Illinois;

4. Department of Behavioral Sciences, Rush University Medical Center,Chicago, Illinois.

Address correspondence to Christopher C. Stewart, Department of Neurology, Indiana University School of Medicine, 355 W. 16th Street, GH4222, Indianapolis, IN 46202. Email: chcstew@iu.edu

This is the author's manuscript of the article published in final edited form as:

Stewart, C. C., Yu, L., Wilson, R. S., Bennett, D. A., \& Boyle, P. A. (2019). Healthcare and Financial Decision Making and Incident Adverse Cognitive Outcomes among Older Adults. Journal of the American Geriatrics Society, 67(8), 1590-1595. https://doi.org/10.1111/jgs. 15880 


\begin{abstract}

\section{OBJECTIVES}

To determine if poorer healthcare and financial decision making forecasts adverse cognitive outcomes in old age. Specifically, we hypothesized that poorer decision making would be associated with an increased risk of incident Alzheimer's dementia, an increased risk of incident mild cognitive impairment $(\mathrm{MCl})$, and a more rapid decline in cognition.
\end{abstract}

\title{
DESIGN
}

An ongoing prospective observational cohort study of aging (the Rush Memory and Aging Project).

\section{SETTING}

The greater Chicago area.

\section{PARTICIPANTS}

A total of 952 community-based older adults without dementia at baseline.

\section{MEASUREMENTS}

Participants completed a measure of healthcare and financial decision making at baseline and underwent annual standardized evaluations to track clinical status and cognitive functions (global cognition, episodic memory, semantic memory, working memory, perceptual speed, and visuospatial ability).

\section{RESULTS}

During up to 9 years of follow-up $(M=5.2 \mathrm{y}$; standard deviation = 2.7), 156 participants developed Alzheimer's dementia (16.4\% of 952), 253 participants developed $\mathrm{MCl}$ (33.2\% of 760$)$, and each cognitive measure declined (all $P \mathrm{~s}<.001$ ). In Cox proportional hazards models adjusted for age, sex, and education, poorer decision making was associated with an increased risk of incident Alzheimer's dementia (hazard ratio $[\mathrm{HR}]=1.17 ; 95 \%$ confidence interval $[\mathrm{Cl}]=1.10$ $1.24 ; P<.001)$ and incident $\mathrm{MCl}(\mathrm{HR}=1.16 ; 95 \% \mathrm{Cl}=1.10-1.22 ; P<.001)$. Further, in linear mixed-effects models, poorer decision making among those who were initially free of cognitive impairment was associated with a more rapid decline in global cognition and four of five specific cognitive domains (all $P \mathrm{~s}<.05$ ).

\section{CONCLUSION}

Our results suggest that poorer healthcare and financial decision making heralds adverse cognitive outcomes in old age.

Key words: aging; Alzheimer's dementia; cognition; decision making; mild cognitive impairment 
Old age is a time when impactful decisions regarding medical plans, retirement funds, estate planning, and many other health and financial issues abound. These decisions are multifaceted and nuanced; they involve comprehension of the decision at hand, weighing the pros and cons of competing options along multiple dimensions, and ultimately selecting the option that is judged best.1- $\underline{3}$ Unfortunately, older adults often make poor healthcare and financial decisions, $\underline{\mathbf{1}}, \underline{\mathbf{4}} \underline{\mathbf{6}}$ and this places them an increased risk of adverse financial and health outcomes including an increased risk of mortality. $\underline{\mathbf{7}}, \underline{\mathbf{8}}$

In addition to its relation with adverse health and financial outcomes, poorer healthcare and financial decision making might be an important antecedent of adverse cognitive outcomes in old age. Healthcare and financial decision making is highly complex, drawing on a diverse set of interrelated factors and resources. For example, using path analysis, we recently demonstrated that about two-thirds of the associations of older age and lower education with poorer decision making works through lower cognition and lower health and financial literacy (ie, the ability to access, understand, and utilize information about health/healthcare and finances). $\underline{9}$ Further, we found that about one-third of the association of lower cognition with poorer decision making works through lower literacy. Additionally, lower literacy and greater risk aversion (ie, one's propensity for overly conservative decisions) were independently associated with poorer decision making. Given this complexity, healthcare and financial decision making might be particularly sensitive to age-related brain changes and therefore may be an early indicator of subsequent adverse cognitive outcomes.

Although no study to our knowledge has investigated the association of decision making with incident adverse cognitive outcomes, prior studies have shown that older adults with mild cognitive impairment $(\mathrm{MCl})$ perform worse on measures of healthcare and financial decision making compared with cognitively intact older adults (ie, no dementia or $\mathrm{MCl}$ ), 1012 and limited evidence indicates that healthcare and financial decision making declines over time in $\mathrm{MCl}, \underline{13}, \underline{14}$ particularly among those who progress to dementia within 1 or 2 years.15, 16 Further, we previously associated a more rapid prior rate of cognitive decline with poorer healthcare and financial decision making among cognitively intact older adults.17 Together, these data suggest that decision making partly reflects subtle age- 
related changes in cognitive function, but studies have not determined whether poorer healthcare and financial decision making predicts the subsequent development of adverse cognitive outcomes. This represents an important gap in knowledge given the need to identify the earliest behavioral manifestations of pathologic cognitive aging. $\underline{\mathbf{1 8}} \underline{\mathbf{2 0}}$

In the current study, we examined associations of healthcare and financial decision making with incident adverse cognitive outcomes among a group of 952 communitybased older adults from the Rush Memory and Aging Project (MAP), a longitudinal clinical-pathologic study of aging.21 Participants were without dementia at baseline, and clinical status and cognition were tracked annually for up to 9 years. We hypothesized that poorer healthcare and financial decision making at baseline would be associated with an increased risk of incident Alzheimer's dementia. Further, given the interest in early signs of pathologic cognitive aging, we hypothesized that poorer decision making would be associated with an increased risk of incident $\mathrm{MCl}$. Additionally, to address the wellestablished association of starting level of cognition with adverse cognitive outcomes, $\underline{22}$ we performed mixed-effects models that simultaneously examined associations of decision making with starting level and rate of cognitive decline, the principal manifestation of Alzheimer's disease, among persons initially free of cognitive impairment. We hypothesized that poorer decision making would be associated with a more rapid decline in cognition after having accounted for the association of decision making with starting level of cognition.

\section{METHODS}

\section{PARTICIPANTS}

Participants were Chicago-area residents enrolled in MAP, an ongoing longitudinal clinical-pathologic study of aging and age-associated diseases.21 Informed consent was obtained from participants following a discussion of the benefits and risks of study participation. This study was approved by the institutional review board of Rush University Medical Center.

MAP started in 1997, and the decision-making assessment began in 2010. At the time of the current analyses, 3177 participants had completed the baseline evaluation, but 1078 
had died, 67 had withdrawn before the start of the decision-making assessment, and 78 had moved out of the area or were ineligible due to severe comprehension or sensory limitations. Of the remaining 1954 participants, 125 declined to complete the decisionmaking assessment, 3 completed the decision-making assessment but had not yet received a clinical diagnosis, and 680 had not yet been administered the decision-making assessment. Among the remaining 1146 eligible participants who had completed the decision-making assessment, 62 were diagnosed with dementia, and 35 had missing data. We also excluded 97 participants who had completed the baseline decision-making assessment but had not yet had a follow-up examination. The remaining 952 participants completed at least one follow-up examination and were available for the current analyses.

\section{CLINICAL CLASSIFICATION}

Participants underwent annual standardized evaluations that included a structured medical history, neurologic examination, and a battery of cognitive measures. Per the criteria of the National Institute of Neurological and Communicative Diseases and StrokeAlzheimer's Disease and Related Disorders, a diagnosis of Alzheimer's dementia was made based on a history of cognitive decline and impairment in memory and at least one other cognitive domain.23 Participants with cognitive impairment but who did not meet criteria for dementia were diagnosed with $\mathrm{MCl} . \underline{24}$

\section{ASSESSMENT OF HEALTHCARE AND FINANCIAL DECISION MAKING}

Healthcare and financial decision making was measured via a modified 12-item version of a well-validated but more extensive and time-consuming healthcare and financial decision-making instrument. $\underline{\mathbf{1}} \underline{\mathbf{3}}, \underline{\mathbf{9}}, \underline{\mathbf{2 5}}$ Our modified version consisted of two 6-item healthcare and financial modules, and each module consisted of three simple and three complex items. Mimicking the decisions older adults often face, participants viewed tables showing information about health maintenance organization plans and mutual funds and then answered questions that required comprehension and integration of the tabulated information. Simple items contained less information in the tables and fewer answer choices to select from. For example, one of the simple financial items consisted of a table showing four characteristics of three mutual funds (gross annual return, management fee, 
minimum investment, and years of activity), and participants were instructed to select the mutual fund that had a management fee of less than $1 \%$ and a gross annual return of over $6.5 \%$. Correspondingly, one of the complex financial items consisted of a table showing five characteristics of seven mutual funds (the four prior characteristics plus percentage of asset allocation to bonds), and participants were asked to select the mutual fund that would provide the best net rate of return after subtracting management fees. Performance was quantified as the total number of items answered correctly (range $=0$ 12). The instrument took less than 15 minutes to complete (typically $<10 \mathrm{~min}$ ). The original decision-making measure was shown to have adequate interrater reliability and shortterm temporal stability. $\underline{\mathbf{1}}, \underline{\mathbf{3}}$ The current 12 -item version had adequate internal consistency among the current group of participants (Cronbach's coefficient $\alpha=.75$ ). There was no evidence of floor or ceiling effects in the current group as only $3.5 \%$ of participants responded incorrectly on all items, and only $2.7 \%$ of participants responded correctly on all items. Also, we previously associated performance on the decision-making measure with relevant decision-making resources (education, cognition, health and financial literacy, risk aversion) and an adverse outcome (mortality), $\underline{\mathbf{7}}, \underline{\mathbf{9}}$ which speaks to the instrument's validity and predictive value.

\section{ASSESSMENT OF COGNITION}

As part of their annual evaluation, participants completed a standardized battery of 19 performance-based cognitive tests. From this, composite indices of global cognition and five specific cognitive domains were calculated.24 The five specific cognitive domains were episodic memory, semantic memory, working memory, perceptual speed, and visuospatial ability. Raw scores on the 19 individual tests were transformed to $z$ scores using the baseline mean and standard deviation (SD) of the entire MAP cohort. Global cognition was calculated by averaging the $z$ scores of all 19 tests, and composite scores of the five specific cognitive domains were calculating by averaging the $z$ scores of individual tests from their respective cognitive domain.

\section{STATISTICAL ANALYSIS}


Bivariate correlations of healthcare and financial decision making with age, sex, education, cognition at baseline, and clinical status at baseline (ie, cognitively intact or $\mathrm{MCl}$ ) were examined via $t$ tests, Wilcoxon rank sum tests, or Pearson correlations, as appropriate. Associations of decision making with incident Alzheimer's dementia and incident $\mathrm{MCl}$ were examined via separate proportional hazards models that adjusted for age at baseline, sex, and education. We also performed a series of mixed-effects analyses that simultaneously modeled the associations of decision making with the starting level and rate of cognitive decline among persons initially free of cognitive impairment. These models consisted of a term for decision making, time in years since baseline, and the interaction of decision making with time. Also included in the models were terms for age, sex, and education, plus their interactions with time. We first ran a model with global cognition as the outcome followed by separate models with each of the five specific cognitive domains as outcomes.

\section{RESULTS}

Baseline characteristics of the group are displayed in Table 1. Mean performance on the decision-making measure was $7.6(63.3 \%$ correct) $(S D=2.8)$. Poorer decision making was associated with older age $(r=-.33 ; P<.001)$, lower education $(r=.38 ; P<.001)$, and poorer cognition (all $P \mathrm{~s}<.001$; Table $\underline{\mathbf{S 1}}$ ). Decision-making scores were lower among women than men $\left(M_{\text {women }}=7.4, S D_{\text {women }}=2.8 ; M_{\text {men }}=8.2, S D_{\text {men }}=2.6 ; t=-3.5 ; P<.001\right)$. At baseline, 760 participants were classified as cognitively intact ( $80 \%$ of 952$)$. Decision making was poorer among participants with $\mathrm{MCl}$ compared with those who were cognitively intact $\left(M_{\mathrm{MCl}}=6.4, S D_{\mathrm{MCl}}=2.8 ; M_{\text {Intact }}=7.9, S D_{\text {Intact }}=2.7 ; t=-7.0 ; P<.001\right)$, consistent with our prior findings.11

Table 1. Characteristics of the Group

Age at baseline, $y$

$80.8(7.3)$ 
Female, \%

Education, y

MMSE at baseline, $0-30$

Decision making, 0-12
$76.6 \%$

$15.4(3.0)$

$28.2(1.79)$

$7.6(2.8)$

Values are mean (standard deviation).

Abbreviation: MMSE, Mini-Mental State Examination.

\section{ASSOCIATIONS OF HEALTHCARE AND FINANCIAL DECISION MAKING WITH INCIDENT ADVERSE COGNITIVE OUTCOMES}

During up to 9 years of follow-up ( $M=5.2 \mathrm{y}$; $S D=2.7), 156$ participants developed Alzheimer's dementia (16.4\% of 952 ). In a proportional hazards model adjusted for age, sex, and education, poorer decision making was associated with an increased risk of incident Alzheimer's dementia (hazard ratio $[H R]=1.17 ; 95 \%$ confidence interval $[\mathrm{Cl}]=1.10-1.24 ; P<.001)$. As illustrated in Figure 1, a participant with poor decision making (score $=4,33 \%$ correct, 10th percentile) was about three times more likely to develop Alzheimer's dementia compared with a participant with good decision making (score $=11,92 \%$ correct, 90th percentile.)

Fig. 1 


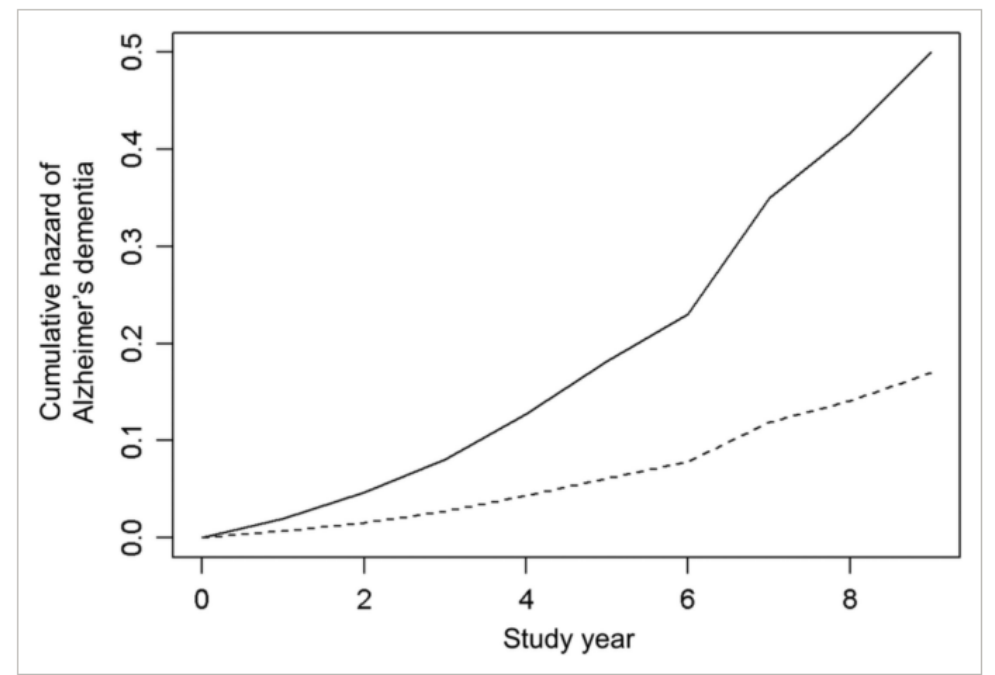

Cumulative hazard of Alzheimer's dementia for participants with poor (solid line, 10th percentile) vs good (dashed line, 90th percentile) decision making, adjusted for age, sex, and education.

Next, because we were interested in the earliest manifestations of pathologic cognitive aging, we examined the association of decision making with incident $\mathrm{MCl}$ among persons initially free of any cognitive impairment (ie, no dementia or $\mathrm{MCl}$ at baseline) $(n=760)$. Progression to $\mathrm{MCl}$ occurred in 253 participants during the follow-up period (33.2\% of 760). After adjusting for age, sex, and education, poorer decision making was associated with an increased risk of incident $\mathrm{MCl}(\mathrm{HR}=1.16 ; 95 \% \mathrm{Cl}=1.10-1.22 ; P<.001)$, such that a participant with poor decision making (score $=4,33 \%$ correct, 10 th percentile) was approximately 2.8 times more likely to develop $\mathrm{MCl}$ compared with a participant with good decision making (score $=11,92 \%$ correct, 90th percentile) (Figure 2 ).

Fig. 2 


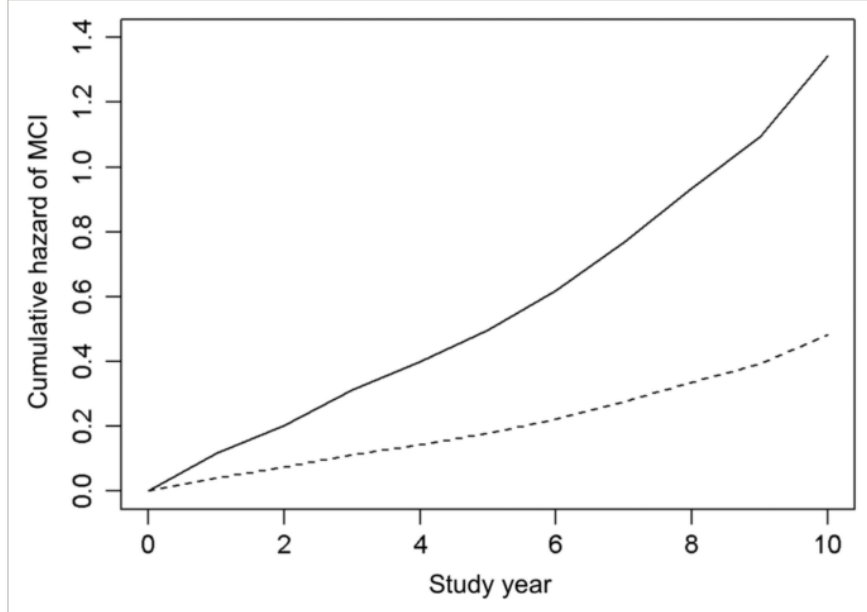

Cumulative hazard of mild cognitive impairment $(\mathrm{MCl})$ for participants with poor (solid line, 10th percentile) vs good (dashed line, 90th percentile) decision making, adjusted for age, sex, and education.

Finally, we examined associations of decision making with starting level and rate of cognitive decline among older adults initially free of cognitive impairment. Performance on each cognitive measure declined during the follow-up period (all $P \mathrm{~s}<.001$ ). In a linear mixed-effects model adjusted for age, sex, and education, poorer decision making was associated with lower global cognition at baseline (estimate $=-.067$; standard error $[\mathrm{SE}]=.005 ; P<.001)$ and a more rapid decline in global cognition over time (estimate $=-.0071 ; \mathrm{SE}=.001 ; P<.001)($ Figure $\underline{3})$. Similar results were observed in the models examining the five cognitive domains. Specifically, poorer decision making was associated with lower baseline performance in all five cognitive domains (all $P \mathrm{~s}<.001$ ) and a more rapid decline in four of five specific cognitive domains (all $P \mathrm{~s}<.05$ ), with the exception of decline in processing speed $(P=.07)$ (Table $\underline{\mathbf{2}}$ ).

\section{Fig. 3}




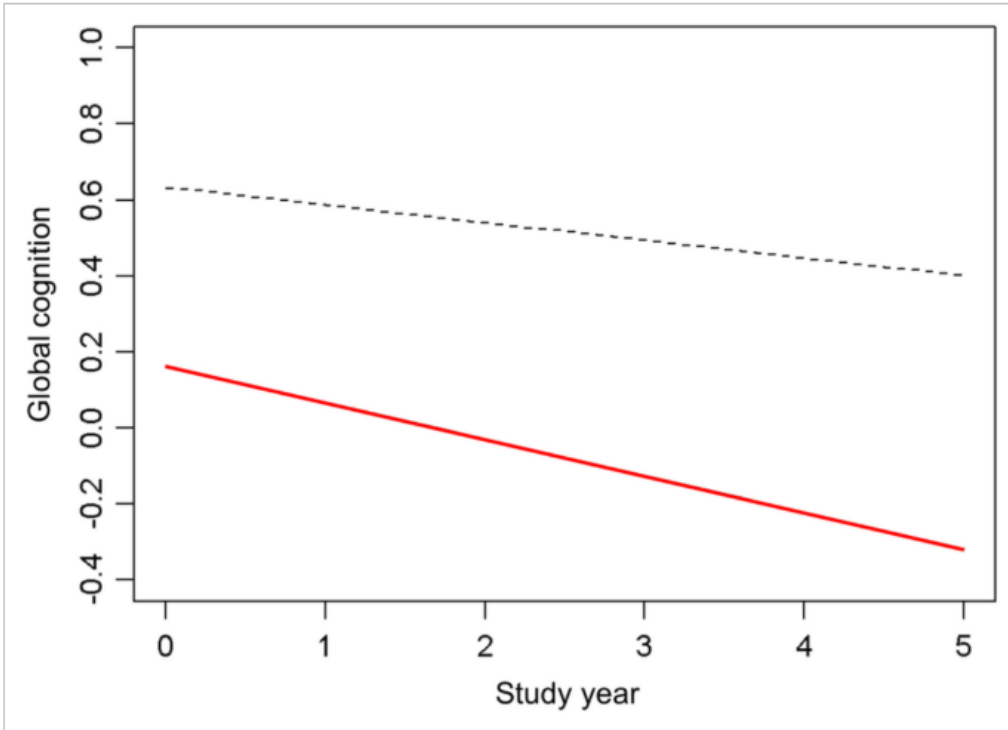

Predicted starting level and 5-year paths of change in global cognition in persons with poor (red line, 10th percentile) vs good (dashed line, 90th percentile) among participants with no cognitive impairment at baseline.

Table 2. Associations of Decision Making with Baseline Cognition and Cognitive Decline Among Older Adults with No Cognitive Impairment at Baseline

\begin{tabular}{lllll|} 
Outcome & Model term & Estimate & SE & $P$ value \\
Global cognition & Decision making & .067 & .005 & $<.001$ \\
& Time & -.125 & .014 & $<.001$ \\
& Decision making $\times$ time & -.007 & .001 & $<.001$ \\
Episodic memory & Decision making & .047 & .006 & $<.001$ \\
& Time & -.125 & .018 & $<.001$ \\
& Decision making $\times$ time & -.008 & .002 & $<.001$ \\
\hline
\end{tabular}




\begin{tabular}{|c|c|c|c|c|}
\hline Outcome & Model term & Estimate & SE & $P$ value \\
\hline \multirow[t]{3}{*}{ Semantic memory } & Decision making & .074 & .006 & $<.001$ \\
\hline & Time & -.118 & .014 & $<.001$ \\
\hline & Decision making $\times$ time & -.007 & .001 & $<.001$ \\
\hline \multirow[t]{3}{*}{ Working memory } & Decision making & .077 & .009 & $<.001$ \\
\hline & Time & -.089 & .015 & $<.001$ \\
\hline & Decision making $\times$ time & -.005 & .001 & $<.01$ \\
\hline \multirow[t]{3}{*}{ Perceptual speed } & Decision making & .098 & .008 & $<.001$ \\
\hline & Time & -.128 & .015 & $<.001$ \\
\hline & Decision making $\times$ time & -.003 & .001 & .070 \\
\hline \multirow[t]{3}{*}{ Visuospatial ability } & Decision making & .086 & .008 & $<.001$ \\
\hline & Time & -.066 & .014 & $<.001$ \\
\hline & Decision making $\times$ time & -.004 & .001 & .015 \\
\hline
\end{tabular}

From separate mixed-effects models adjusted for age, sex, and education, plus their interactions with time.

Abbreviation: SE, standard error.

DISCUSSION 
In the present study, we examined associations of healthcare and financial decision making with incident adverse cognitive outcomes among a group of more than 900 older adults, all of whom were without dementia at baseline. During up to 9 years of follow-up, we found that poorer decision making was associated with a substantially increased risk of incident Alzheimer's dementia. Further, among persons initially free of any cognitive impairment (ie, no dementia or $\mathrm{MCl}$ ), we found that poorer decision making was associated with an increased risk of incident $\mathrm{MCl}$ and a more rapid decline in global cognition, episodic memory, semantic memory, working memory, and visuospatial ability. Our results suggest that poorer healthcare and financial decision making is a harbinger of adverse cognitive outcomes in old age.

Prior research examining healthcare and financial decision making in aging has generally focused on individuals with overt cognitive syndromes such as $\mathrm{MCl}$. These studies showed that performance on measures of healthcare and financial decision making is poorer among older adults with $\mathrm{MCl}$ compared with cognitively intact older adults.1012 They also suggest that decision making declines over time in $\mathrm{MCl}, \underline{13}, \underline{14}$ especially among those who progress to dementia within 1 or 2 years.15, 16 Although this literature establishes associations of decision making with clinical status and progression, it does not address the question of whether poorer decision making is a by-product of adverse cognitive outcomes or a bellwether of said outcomes. The current findings provide clear support for the latter. Poorer decision making was not only related to incident Alzheimer's dementia and incident $\mathrm{MCl}$ but also with a more rapid cognitive decline among older adults who were initially free of cognitive impairment. Importantly, the association of poorer decision making with cognitive decline was relatively independent of starting level of cognition, suggesting that the relation of decision making with decline was not merely due to its association with a lower level of cognition at baseline.

The reason why poorer healthcare and financial decision making is associated with incidence adverse cognitive outcomes is not clear, but we suspect that Alzheimer's disease pathology and other common age-related neuropathologies play an important role. As discussed at the outset, healthcare and financial decision making is highly complex, and good decision making depends on the interplay between diverse factors 
and resources including age, education, cognition, health and financial literacy, and aspects of decision-making style (eg, risk aversion, temporal discounting).1-ㅗㄴ $\underline{\mathbf{9}}$ Poor decision making occurs when this network of factors and resources is deficient or disrupted in some manner. Given the complexity involved and the multiple opportunities for breakdowns, healthcare and financial decision making might be particularly sensitive to the subclinical accumulation of Alzheimer's disease pathology, and this might explain why poorer decision making, even among cognitively intact older adults, forecasts incident adverse cognitive outcomes. The notion that decision making declines in relation to the early buildup of Alzheimer's disease pathology is also consistent with our previous study showing that a more rapid prior rate of cognitive decline is associated with poorer healthcare and financial decision among cognitively intact older adults. $\underline{17}$

In addition to implicating healthcare and financial decision making in the development of adverse cognitive outcomes, the present study underscores the potential utility of instruments assessing higher-order functional abilities and our measure of decision making specifically. Conventional thinking holds that functional decline is a relatively late manifestation of Alzheimer's disease; however, some have suggested that the time course of functional decline depends on the ability under investigation and how it is measured. For example, Marson 19 recommended that functional instruments should assess complex abilities that are central to independent living and should be performance-based and use an interval scale to capture nuanced information about the ability. The current healthcare and financial decision making measure, which was modified from a well-validated but more time-consuming decision-making instrument, $\underline{\mathbf{1}}, \underline{\mathbf{3}}$ incorporates these features. As discussed earlier, we suspect that the complexity of healthcare and financial decision making is central to why it predicted adverse cognitive outcomes; however, our instrument also is performance based and uses an interval scale, and these characteristics are undoubtedly important as well. Although much work remains, the present findings suggest that the current decisionmaking measure might someday be used clinically as a complement to cognitive screeners or in clinical trials as a supplementary outcome alongside traditional cognitive measures. 
This study has strengths and weaknesses, and it also highlights avenues for future research. A study strength includes the large number of participants in our group and the duration of our follow-up period, both of which are substantial improvements over existing studies investigating healthcare and financial decision making in relation to clinical status and progression. A study weakness is that our study group was predominantly white $(95 \%)$ and relatively highly educated. Given the well-established disparities in health and healthcare among historically underserved groups, $\underline{\mathbf{2 6}}$ it will be important to determine if the current findings generalize to older adults who are racial minorities or have lower educational attainment. We are collecting decision-making data among these groups and will determine if the present findings generalize once we have sufficient data. Future research also should aim to elucidate the neural and neuropathologic pathways underlying the association of decision making with incident adverse cognitive outcomes, as this work might ultimately aid in the early detection of neuropathology.

\section{ACKNOWLEDGMENTS}

We extend our heartfelt thanks to the thousands of participants in the Rush Memory and Aging Project and the staff of the Rush Alzheimer's Disease Center (RADC). This work would not be possible without their efforts. To request data from the Rush Memory and Aging Project for research purposes, visit the RADC Research Resource Sharing Hub (www.radc.rush.edu).

Financial Disclosure : The study was supported by National Institute on Aging grants R01AG017917 (Bennett), R01AG033678 (Boyle), R01AG034374 (Boyle), and R01AG060376 (Boyle).

Conflict of Interest : The authors have no conflicts of interest to disclose in relation to this article.

Author Contributions: Study conceptualization, data analysis, interpretation, and drafting of the manuscript: Stewart, Yu, and Boyle. Study conceptualization, study design, data acquisition and interpretation of findings, and critical review of the manuscript: Bennett and Wilson.

Sponsor's Role : None. 


\section{REFERENCES}

1. Finucane, M. L., \& Gullion, C. M. (2010). Developing a tool for measuring the decisionmaking competence of older adults. Psychology and Aging, 25(2), 271-288. https://doi.org/10.1037/a0019106

2. Li, Y., Baldassi, M., Johnson, E. J., \& Weber, E. U. (2013). Complementary cognitive capabilities, economic decision making, and aging. Psychology and Aging, 28(3), 595613. https://doi.org/10.1037/a0034172

3. Finucane, M. L., Mertz, C. K., Slovic, P., \& Schmidt, E. S. (2005). Task Complexity and Older Adults' Decision-Making Competence. Psychology and Aging, 20(1), 71-84. https://doi.org/10.1037/0882-7974.20.1.71

4. Amalraj, S., Starkweather, C., Nguyen, C., \& Naeim, A. (2009). Health literacy, communication, and treatment decision-making in older cancer patients. Oncology, 23(4), 369-369.

5. Chesser, A. K., Keene Woods, N., Smothers, K., \& Rogers, N. (2016). Health Literacy and Older Adults: A Systematic Review. Gerontology and Geriatric Medicine, 2, 2333721416630492. https://doi.org/10.1177/2333721416630492

6. Lusardi, A., \& Mitchell, O. S. (2011). Financial Literacy and Planning: Implications for Retirement Wellbeing (Working Paper No. 17078; Working Paper Series). National Bureau of Economic Research. https://doi.org/10.3386/w17078

7. Boyle, P. A., Wilson, R. S., Yu, L., Buchman, A. S., \& Bennett, D. A. (2013). Poor Decision Making Is Associated with an Increased Risk of Mortality among Community-Dwelling Older Persons without Dementia. Neuroepidemiology, 40(4), 247-252. https://doi.org/10.1159/000342781

8. Lusardi, A., \& Mitchell, O. S. (2014). The Economic Importance of Financial Literacy: Theory and Evidence. Journal of Economic Literature, 52(1), 5-44. https://doi.org/10.1257/jel.52.1.5

9. Stewart, C. C., Yu, L., Wilson, R. S., Bennett, D. A., \& Boyle, P. A. (2018). Correlates of healthcare and financial decision making among older adults without dementia. Health Psychology, 37(7), 618-626. https://doi.org/10.1037/hea0000610 
10. Griffith, H. R., Belue, K., Sicola, A., Krzywanski, S., Zamrini, E., Harrell, L., \& Marson, D. C. (2003). Impaired financial abilities in mild cognitive impairment: A direct assessment approach. Neurology, 60(3), 449-457. https://doi.org/10.1212NWNL.60.3.449

11. Han, S. D., Boyle, P. A., James, B. D., Yu, L., \& Bennett, D. A. (2015). Mild Cognitive Impairment Is Associated with Poorer Decision-Making in Community-Based Older Persons. Journal of the American Geriatrics Society, 63(4), 676-683. https://doi.org/10.1111/jgs.13346

12. Okonkwo, O., Griffith, H., Belue, K., Lanza, S., Zamrini, E., Harrell, L., Brockington, J., Clark, D., Raman, R., \& Marson, D. (2007). Medical decision-making capacity in patients with mild cognitive impairment. Neurology, 69(15), 1528-1535. https://doi.org/10.1212/01.wnl.0000277639.90611.d9

13. Martin, R. C., Gerstenecker, A., Triebel, K. L., Falola, M., McPherson, T., Cutter, G., \& Marson, D. C. (2019). Declining Financial Capacity in Mild Cognitive Impairment: A SixYear Longitudinal Study. Archives of Clinical Neuropsychology, 34(2), 152-161. https://doi.org/10.1093/arclin/acy030

14. Okonkwo, O., Griffith, H., Copeland, J., Belue, K., Lanza, S., Zamrini, E., Harrell, L., Brockington, J., Clark, D., Raman, R., \& Marson, D. (2008). Medical decision-making capacity in mild cognitive impairment: A 3-year longitudinal study. Neurology, 71(19), 1474-1480. https://doi.org/10.1212/01.wnl.0000334301.32358.48

15. Gerstenecker, A., Triebel, K. L., Martin, R., Snyder, S., \& Marson, D. C. (2016). Both Financial and Cognitive Decline Predict Clinical Progression in MCl. Alzheimer Disease \& Associated Disorders, 30(1), 27-34. https://doi.org/10.1097NAD.0000000000000120

16. Triebel, K., Martin, R., Griffith, H., Marceaux, J., Okonkwo, O., Harrell, L., Clark, D., Brockington, J., Bartolucci, A., \& Marson, D. (2009). Declining financial capacity in mild cognitive impairment: A 1-year longitudinal study. Neurology, 73(12), 928-934. https://doi.org/10.1212/WNL.0b013e3181b87971

17. Boyle, P. A., Yu, L., Wilson, R. S., Gamble, K., Buchman, A. S., \& Bennett, D. A. (2012). Poor Decision Making Is a Consequence of Cognitive Decline among Older Persons without Alzheimer's Disease or Mild Cognitive Impairment. PLOS ONE, 7(8), e43647e43647. 
18. Jack Jr., C. R., Bennett, D. A., Blennow, K., Carrillo, M. C., Dunn, B., Haeberlein, S. B., Holtzman, D. M., Jagust, W., Jessen, F., Karlawish, J., Liu, E., Molinuevo, J. L., Montine, T., Phelps, C., Rankin, K. P., Rowe, C. C., Scheltens, P., Siemers, E., Snyder, H. M., .. Silverberg, N. (2018). NIA-AA Research Framework: Toward a biological definition of Alzheimer's disease. Alzheimer's \& Dementia, 14(4), 535-562. https://doi.org/10.1016/j.jalz.2018.02.018

19. Marson, D. (2015). Investigating Functional Impairment in Preclinical Alzheimer's Disease. The Journal of Prevention of Alzheimer's Disease, 2(1), 4-6. https://doi.org/10.14283/jpad.2015.44

20.Sperling, R. A., Aisen, P. S., Beckett, L. A., Bennett, D. A., Craft, S., Fagan, A. M., Iwatsubo, T., Jack, C. R., Kaye, J., Montine, T. J., Park, D. C., Reiman, E. M., Rowe, C. C., Siemers, E., Stern, Y., Yaffe, K., Carrillo, M. C., Thies, B., Morrison-Bogorad, M., ... Phelps, C. H. (2011). Toward defining the preclinical stages of Alzheimer's disease: Recommendations from the National Institute on Aging-Alzheimer's Association workgroups on diagnostic guidelines for Alzheimer's disease. Alzheimer's \& Dementia, 7(3), 280-292. https://doi.org/10.1016/j.jalz.2011.03.003

21. Bennett, D. A., Buchman, A. S., Boyle, P. A., Barnes, L. L., Wilson, R. S., \& Schneider, J. A. (2018). Religious Orders Study and Rush Memory and Aging Project. Journal of Alzheimer's Disease : JAD, 64(Suppl 1), S161-S189. https://doi.org/10.3233/JAD-179939 22. Knopman, D., Beiser, A., Machulda, M., Fields, J., Roberts, R., Pankratz, V., Aakre, J., Cha, R., Rocca, W., Mielke, M., Boeve, B., Devine, S., Ivnik, R., Au, R., Auerbach, S., Wolf, P., Seshadri, S., \& Petersen, R. (2015). Spectrum of cognition short of dementia: Framingham Heart Study and Mayo Clinic Study of Aging. Neurology, 85(19), 1712-1721. https://doi.org/10.1212/WNL.0000000000002100

23. McKhann, G., Drachman, D., Folstein, M., Katzman, R., Price, D., \& Stadlan, E. (1984). Clinical diagnosis of Alzheimer's disease: Report of the NINCDS-ADRDA Work Group* under the auspices of Department of Health and Human Services Task Force on Alzheimer's Disease. Neurology, 34(7), 939-944.

24. A. Bennett, D., A. Schneider, J., S. Buchman, A., L. Barnes, L., A. Boyle, P., \& S. Wilson, R. (2012). Overview and Findings from the Rush Memory and Aging Project. Current Alzheimer Research, 9(6), 646-663. https://doi.org/10.2174/156720512801322663 
25. A. Bennett, D., A. Schneider, J., S. Buchman, A., L. Barnes, L., A. Boyle, P., \& S. Wilson, R. (2012). Overview and Findings from the Rush Memory and Aging Project. Current Alzheimer Research, 9(6), 646-663. https://doi.org/10.2174/156720512801322663

26. Wheeler, S. M., \& Bryant, A. S. (2017). Racial and Ethnic Disparities in Health and Health Care. Obstetrics and Gynecology Clinics, 44(1), 1-11. https://doi.org/10.1016/j.ogc.2016.10.001 